\title{
POSTULATES FOR GENERALIZATIONS OF HILBERT SPACE
}

\author{
H. RUBIN AND M. H. STONE
}

If the requirements affecting dimensionality and completeness are suppressed from von Neumann's well known system of postulates for complex abstract Hilbert space [2], the remaining postulates focus attention on a complex linear space (or complex module) specialized by the demand that it carry a positive strictly definite, Hermitian symmetric, bilinear form. Jordan and von Neumann [1] have shown that such a linear space can equally well be characterized postulationally as a normed complex linear space in which the norm satisfies a simple identity (see (14) below) expressing in vector language the following geometrical property of the parallelogram, verified in euclidean spaces: the sum of the squares of the diagonals is equal to the sum of the squares of the four sides. It is natural to inquire whether the Jordan-von Neumann postulates cannot be simplified by showing that the characteristic properties of a norm (in particular, the triangle inequality) can be derived from the parallelogram identity, reinforced only by the weakest of additional assumptions. We shall show here that this is indeed so, including in our discussion not only the complex case but also the real and the quaternionic cases. Thus the principal demand which we shall impose for our purposes upon a linear space is that it shall carry a positive real function satisfying a variant (see (1) below) of the parallelogram identity calculated to guarantee its identification as a quadratic or Hermitian form. Observations along these lines have surely been made by other mathematicians interested in Hilbert space; but so far as we know none has yet been recorded in the standard literature. ${ }^{1}$

We start from the following basic postulates:

Postulate 1. $X$ is a linear space (or module), with elements $x, y, z, \cdots$, over the real, complex, or quaternionic number system $\Phi$, with elements $\alpha, \beta, \gamma, \cdots .^{2}$

Postulate 2. On $X$ there is defined a non-negative real function $q$ 1952.

Presented to the Society, October 29, 1949; received by the editors December 8 ,

${ }^{1}$ In lectures delivered in the Autumn of 1948 and later distributed privately in mimeographed form, N. Aronszajn has indeed made similar observations. Our own work on these questions dates from the winter of 1947.

2 The zero element of $X$ will be denoted as $\theta$, that of $\Phi$ as 0 ; and the conjugate of a complex number or quaternion $\alpha$ will be denoted as $\alpha^{*}$. 
such that

$$
q(x+y)+q(x-y)=2 q(x)+2 q(y) .
$$

Postulate 3. As a function of the real number $\alpha$ the quantity $q(\alpha x)$ is bounded in some neighborhood of $\alpha=0$ for each $x$.

Postulate 4. In the complex and quaternionic cases the relations $q(x)=q(i x), q(x)=q(i x)=q(j x)=q(k x)$ hold for the imaginary units $i$ and $i, j, k$, respectively.

We remark that our analysis will disclose the existence of possible simple substitutes for Postulate 3; and also that Postulate 4 can be replaced by the apparently stronger requirement that $|\alpha|=|\beta|$ imply $q(\alpha x)=q(\beta x)$, a requirement which makes no distinction between the real, complex, and quaternionic cases. If it is desired, our list of postulates can be augmented by adding:

Postulate 5. If $q(x)=0$ then $x=\theta$.

However, we shall proceed without relying on this assumption, merely noting at the end of the discussion the simple consequences which follow from it.

We introduce a real function $p$ of two arguments by putting

$$
4 p(x, y)=q(x+y)-q(x-y)
$$

and then make the

Definition 1. The quantity $x y \in \Phi$ given by the respective relations

$$
\begin{aligned}
& x y=p(x, y), \\
& x y=p(x, y)+i p(x, i y), \\
& x y=p(x, y)+i p(x, i y)+j p(x, j y)+k p(x, k y),
\end{aligned}
$$

according as $\Phi$ is the real, complex, or quaternionic number system, is called the scalar product in $X$.

We now have:

THEOREM 1a. In the real case the scalar product $x y=p(x, y)$ is symmetric, linear in both arguments, and positive definite (in the sense that $x x=q(x) \geqq 0$ for all $x$ in $X)$. It obeys the Schwarz inequality, in the form $|x y|^{2} \leqq(x x)(y y)$.

Proof. We first prove some simple properties of $q$, based on the identity (1) and independent of the nature of $\Phi$. If we put $x=y=\theta$ in (1), we obtain $q(\theta)=0$; if we then put $y=x$ in (1) we obtain $q(2 x)$ $=4 q(x)$; and if we put $x=\theta$ in (1) we obtain $q(-y)=q(y)$. Applying these results in (2), (3), (4), (5) we see that $(-x) y=-(x y), \theta y=0$ in all cases. In the real cases we see that $x x=p(x, x)=q(x) \geqq 0$, and that 
$x y=y x$. It is slightly more difficult to show that $x y$ is additive in its first argument (and hence also in it second). Using (1), we write with Jordan and von Neumann [1]

$$
\begin{aligned}
q\left(\left(x+x^{\prime}\right)+y\right)= & q\left((x+y / 2)+\left(x^{\prime}+y / 2\right)\right) \\
= & 2 q(x+y / 2)+2 q\left(x^{\prime}+y / 2\right) \\
& -q\left((x+y / 2)-\left(x^{\prime}+y / 2\right)\right) \\
= & 2 q(x+y / 2)+2 q\left(x^{\prime}+y / 2\right)-q\left(x-x^{\prime}\right), \\
q\left(\left(x+x^{\prime}\right)-y\right)= & 2 q(x-y / 2)+2 q\left(x^{\prime}-y / 2\right)-q\left(x-x^{\prime}\right) .
\end{aligned}
$$

Subtracting the second equation from the first and applying (2) and (3) we obtain

$$
\begin{aligned}
4\left(\left(x+x^{\prime}\right) y\right)= & 2(q(x+y / 2)-q(x-y / 2)) \\
& +2\left(q\left(x^{\prime}+y / 2\right)-q\left(x^{\prime}-y / 2\right)\right) \\
= & 8(x(y / 2))+8\left(x^{\prime}(y / 2)\right) .
\end{aligned}
$$

With $x^{\prime}=\theta$, this identity yields $x y=2(x(y / 2))$ and therefore must itself assume the form

$$
\left(x+x^{\prime}\right) y=x y+x^{\prime} y,
$$

as we wished to prove. The relations $(6),(-x) y=-(x y)$, and $\theta y=0$ imply, as is well known, that the relation

$$
(\alpha x) y=\alpha(x y)
$$

is valid for all rational $\alpha$. Actually we can remove the restriction on $\alpha$. To do so we first establish the Schwarz inequality. Using (6), (7), and the symmetry of the scalar product we can write

$$
\alpha^{2}(x x)+2 \alpha \beta(x y)+\beta^{2}(y y)=(\alpha x+\beta y)(\alpha x+\beta y) \geqq 0
$$

for arbitrary rational numbers $\alpha$ and $\beta$. By continuity the quadratic form on the left must therefore be non-negative for all real $\alpha$ and $\beta$. Thus if we put $\alpha=-x y, \beta=x x$ we find that $(x x)\left(-|x y|^{2}+(x x)(y y)\right)$ $\geqq 0$. Thus we conclude that

$$
|x y|^{2} \leqq(x x)(y y)
$$

except possibly in the case where $x x=0$. We can show, however, that $x x=0$ implies $x y=0$ and hence gives rise to no exception in (8). For this purpose it suffices for us to put $\beta=x y$ above and to choose $\alpha$ so that $2 \alpha+y y<0$, obtaining $(2 \alpha+y y)|x y|^{2} \geqq 0, x y=0$. Combining (8) and Postulate 3, we now see that as a function of $\alpha$ the quantity $\phi(\alpha)=(\alpha x) y$ is bounded in a neighborhood of $\alpha=0$. From (6) we note that 


$$
\phi(\alpha+\beta)=\phi(\alpha)+\phi(\beta)
$$

since $((\alpha+\beta) x) y=(\alpha x+\beta x) y=(\alpha x) y+(\beta x) y$. The classical theory [3] of the functional equation (9) shows that $\phi(\alpha)=\alpha \phi(1)$-that is, that (7) is valid for all real $\alpha$. This completes the proof.

We may now remark, before proceeding to the other cases, that any condition on $q$ which will guarantee the desired determination of $\phi(\alpha)$ from the functional equation (9) will serve our purposes as well as Postulate 3 has done. For example it would be sufficient [4] to require that $q(\alpha x+y)$ be a measurable function of the real number $\alpha$ for every pair $(x, y)$, since $\phi(\alpha)$ would then be measurable also in accordance with (2) and (3). Turning now to the next case, we have

TheOREM $1 \mathrm{~b}$. In the complex case the scalar product $x y$ is Hermitian symmetric (in the sense that $y x=(x y)^{*}$ ), linear in its first argument, anti-linear in its second (in the sense that $x\left(\alpha_{1} y_{1}+\alpha_{2} y_{2}\right)=\alpha_{1}^{*}\left(x y_{1}\right)$ $+\alpha_{2}^{*}\left(x y_{2}\right)$ ), and positive definite (in the sense that $x x=q(x) \geqq 0$ for all $x$ in $X)$. It obeys the Schwarz inequality, in the form $|x y|^{2} \leqq(x x)(y y)$.

Proof. By restricting the scalars to be real, we may treat the complex linear space $X$ as real linear. The function $p(x, y)$ therefore has on $X$ all those properties established in Theorem 1a. From Postulate 4 we have accordingly

$$
\begin{aligned}
4 p(i x, y) & =q(i x+y)-q(i x-y) \\
& =q(x-i y)-q(x+i y) \\
& =-4 p(x, i y) .
\end{aligned}
$$

Consequently

$$
\begin{aligned}
y x & =p(y, x)+i p(y, i x)=p(x, y)+i p(i x, y) \\
& =p(x, y)-i p(x, i y)=(x y)^{*} .
\end{aligned}
$$

Since $x x$ is thus seen to be real, it follows that $p(x, i x)=0, x x=p(x, x)$ $=q(x) \geqq 0$. We note that, since $p$ is real linear in each of its arguments, the scalar product $x y$ is also. From the evaluation

$$
\begin{aligned}
(i x) y & =p(i x, y)+i p(i x, i y)=-p(x, i y)-i p(x,-y) \\
& =i p(x, y)-p(x, i y)=i(x y)
\end{aligned}
$$

it is thus easy to derive the fact that $x y$ is complex linear in its first factor. Indeed, if the complex number $\alpha$ has real components $\alpha_{1}, \alpha_{2}$, we have

$$
\begin{aligned}
(\alpha x) y & =\left(\alpha_{1} x+\alpha_{2} i x\right) y=\alpha_{1}(x y)+\alpha_{2}((i x) y) \\
& =\left(\alpha_{1}+\alpha_{2} i\right)(x y)=\alpha(x y) .
\end{aligned}
$$


By virtue of its Hermitian symmetry, the scalar product must then be anti-linear in its second factor. It is now easy to establish the Schwarz inequality. For arbitrary complex numbers we have

$\alpha \alpha^{*}(x x)+\alpha \beta^{*}(x y)+\alpha^{*} \beta(x y)^{*}+\beta \beta^{*}(y y)=(\alpha x+\beta y)(\alpha x+\beta y) \geqq 0$.

If $x x=0$ we take $\beta=x y$ and choose $\alpha$ as a real number such that $2 \alpha$ $+y y<0$. It follows that $(2 \alpha+y y)|x y|^{2} \geqq 0, x y=0,|x y|^{2} \leqq(x x)(y y)$. If $x x>0$ we take $\alpha=-(x y)^{*}, \beta=x x$, obtaining

$$
x x\left(-|x y|^{2}+(x x)(y y)\right)=(\alpha x+\beta y)(\alpha x+\beta y) \geqq 0
$$

and hence $|x y|^{2} \leqq(x x)(y y)$, as we wished to prove.

Finally we have

ThEOREM 1c. In the quaternionic case the scalar product has properties analogous to those established in Theorem $1 \mathrm{~b}$ for the complex case.

The proof is similar to that of Theorem $1 \mathrm{~b}$.

The connection which has thus been established between the function $q$ and the scalar product $x y$ now enables us to introduce a seminorm in $X$ :

THEOREM 2. In all three cases the function defined by the relation $|x|$ $=(q(x))^{1 / 2}=(x x)^{1 / 2}$ is a semi-norm, enjoying the properties:

$$
\begin{aligned}
|x| & \geqq 0, \quad|\theta|=0 ; \\
|\alpha x| & =|\alpha||x| ; \\
|x+y| & \leqq|x|+|y| \\
|x+y|^{2}+|x-y|^{2} & =2|x|^{2}+2|y|^{2} .
\end{aligned}
$$

In terms of it, the Schwarz inequality becomes

$$
|x y| \leqq|x||y| \text {. }
$$

Proof. The properties (11), (14), (15) merely restate previously established properties of $q$ or $x y$; in particular, (14) is a restatement of (1). The relations $|\alpha x|=((\alpha x)(\alpha x))^{1 / 2}=\left(\alpha \alpha^{*}(x x)\right)^{1 / 2}=|\alpha||x|$ yield (12). Finally, (13) is a consequence of the Schwarz inequality since

$$
\begin{aligned}
|x+y|^{2} & =(x+y)(x+y)=x x+x y+y x+y y \\
& \leqq|x|^{2}+2|x y|+|y|^{2} \\
& \leqq|x|^{2}+2|x||y|+|y|^{2}=(|x|+|y|)^{2} .
\end{aligned}
$$

This completes the proof.

It is now easy to discuss the role of Postulate 5. We first establish

Theorem 3. The set $X_{0}=(x ;|x|=0)$ is a linear subspace of $X$, with 
the property that $x-x^{\prime} \in X_{0}$ implies $q(x)=q\left(x^{\prime}\right), x y=x^{\prime} y$. In the Schwarz inequality (15), the sign of equality holds if and only if $x$ and $y$ are linearly dependent (modulo $X_{0}$ ).

Proof. From (11), (12), (13) we see at once that $X_{0}$ is a linear manifold in $X$. It is even closed in the norm topology of $X$ since $x_{n} \rightarrow x$ and $x_{n} \in X_{0}$ imply $|x|=|| x|-| x_{n}|| \leqq\left|x-x_{n}\right| \rightarrow 0,|x|=0$. If $x-x^{\prime}$ $\in X_{0}$ then $|x| \leqq\left|x-x^{\prime}\right|+\left|x^{\prime}\right|=\left|x^{\prime}\right|,\left|x^{\prime}\right| \leqq|x|$, and $q(x)=|x|^{2}$ $=\left|x^{\prime}\right|^{2}=q\left(x^{\prime}\right)$. It is clear from (2), (3), (4), (5) that this result leads also to the conclusion that, if $x-x^{\prime} \in X_{0}$, then $x y=x^{\prime} y$. In discussing the Schwarz inequality we may first take care of the case of equality which occurs when $x x=0$, a case where $x \in X_{0}$ and $x$ and $y$ are trivially linearly dependent (modulo $X_{0}$ ). When $x x>0$ we see that, as in (10),

$$
|x|^{2}\left(-|x y|^{2}+|x|^{2}|y|^{2}\right)=|-(y x) x+(x x) y|^{2} \text {. }
$$

Thus equality now holds in (15) if and only if $-(y x) x+(x x) y \in X_{0}$. This completes the proof.

THEOREM 4. Identification of the elements of $X$ modulo $X_{0}$ and transfer of the function $q$ to the resulting difference space $X-X_{0}$ yields a system obeying Postulate 5 as well as Postulates 1-4. Postulate 5 is equivalent to the requirement that $X_{0}=(\theta)$, and its role is to eliminate any need for the above identification. When Postulate 5 holds, the function $q^{1 / 2}$ introduced in Theorem 2 is a norm, rather than a semi-norm.

Proof. The details are automatic and may be omitted.

\section{REFERENCES}

1. P. Jordan and J. von Neumann, Ann. of Math. (2) vol. 36 (1935) pp. 719-723.

2. J. von Neumann, Math. Ann. vol. 102 (1930) pp. 49-131, especially pp. 64-66.

3. G. Darboux, Math. Ann. vol. 17 (1880) pp. 55-61, especially pp. 56-57, footnote.

4. W. Sierpinski, Fund. Math. vol. 1 (1920) pp. 116-122.

The Univeraity of Chicago 